\title{
Mechanical thrombectomy in patients with proximal occlusions and low NIHSS: Results from a large prospective registry
}

\author{
Leonardo Renieri, MD, ${ }^{*}$ Valentina Saia, MD, + Vitor Mendes Pereira, $\neq$ \\ Giovanni Pracucci, MD,§ Nicola Limbucci, MD,* Sergio Nappini, MD,* \\ Pascal N Tyrrell, $₫$ Francesco Arba, MD,\# Patrizia Nencini, MD,\# \\ Domenico Inzitari,\$ Andrea Giorgianni, MD, ${ }^{* *}$ Massimiliano Natrella, MD,\#\# \\ Roberto Menozzi, MD,\$\$ Umberto Scoditti, MD,\$\$ \\ Salvatore Mangiafico, $\mathrm{MD},{ }^{*}$ and Danilo Toni, $₫ \uparrow$, on behalf of the Italian \\ Registry of Endovascular Treatment in Acute Stroke
}

\begin{abstract}
Background: Mechanical thrombectomy is now standard of care for treatment of acute ischemic stroke secondary to large vessel occlusion in the setting of high NIHSS. We analysed a large nationwide registry focusing on patients with large vessel occlusion and low NIHSS on admission to evaluate the efficacy and safety of thrombectomy in this patient population Methods: 2826 patients treated with mechanical thrombectomy were included in a multicentre registry from January 1, 2011 to December 31, 2015. We included patients with large vessel occlusion and NIHSS $\leq 6$ on admission. Baseline characteristics, imaging, clinical outcome, procedure adverse events and positive and negative outcome predictors were analysed. Results: 134 patients were included. 90/ 134 had an anterior circulation and 44 a posterior circulation stroke. One patient died before treatment. Successful revascularization (mTICI $2 \mathrm{~b}-3$ ) was achieved in $73.7 \%$ $(98 / 133)$ of the patients. Intraprocedural adverse event was observed in $3 \%(4 / 133)$ of cases. Symptomatic intracranial haemorrhage rate was $5.3 \%(7 / 133)$. At three months, $70.9 \%(95 / 134)$ of the patients had $\mathrm{mRS}$ score $0-2,15.7 \%$ (21/134) mRS $3-5$ and $13.4 \%$ $(18 / 134) \mathrm{mRS} 6$. Age and successful recanalization were significant predictors of a good clinical outcome on both univariate $(p=0.005$ and $p=0.007)$ and multivariable $(p=0.0018$ and $p=0.009$ [nat $\log ]$ ) analysis. Absence of vessel recanalization and symptomatic intracranial hemorrhage were independent predictors of poor outcome $(p=0.021)$. Conclusions: Our study suggests that patients with large vessel occlusion and low NIHSS score on admission can benefit from mechanical thrombectomy. Randomized trials are warranted.
\end{abstract}

Keywords: Low NIHSS—Ischemic stroke—Large vessel occlusion—Mechanical thrombectomy

(c) 2020 Elsevier Inc. All rights reserved.

\footnotetext{
From the *Interventional Neurovascular Unit, Careggi University Hospital, Florence, Italy; +Neurology department/Stroke Unit, Santa Corona Hospital, Pietra Ligure, Italy; †Department of Medical Imaging, University Health Network, Toronto, ON, Canada; §Neurofarba Department, University of Florence, Florence, Italy; $\uparrow$ Department of Statistical Sciences, University of Toronto, Toronto, ON, Canada; "Department of Neurology, Careggi University Hospital, Florence, Italy; ${ }^{\$}$ Neurofarba Department, University of Florence, Florence, Italy; ${ }^{* *}$ Neuroradiology Unit, Ospedale di Circolo, Asst-sette Laghi University Hospital, Varese, Italy; "\# Diagnostic and Interventional Radiology Unit, “U. Parini" Valle d'Aosta Regional Hospital, Aosta, Italy; ${ }^{\$ \$}$ Interventional Neuroradiology Unit, University Hospital of Parma, Parma, Italy; §§Stroke Unit, University Hospital of Parma, Parma, Italy; and $₫ \uparrow$ Stroke Emergency Department Stroke Unit, Department of Human Neurosciences, Sapienza University of Rome, Rome, Italy.

Received May 3, 2020; revision received May 29, 2020; accepted June 22, 2020.

Corresponding author. E-mail: leonardo.renieri@hotmail.it.

$1052-3057 / \$$ - see front matter

(c) 2020 Elsevier Inc. All rights reserved.

https://doi.org/10.1016/j.jstrokecerebrovasdis.2020.105091
} 


\section{Introduction}

Endovascular treatment of acute ischemic stroke related to large vessel occlusion has been proven to be safe and effective. ${ }^{1-5}$ Recent randomized controlled trials (RCTs) demonstrated a significant benefit of mechanical thrombectomy compared to IV rtPA or best medical treatment. ${ }^{1-3,6-8}$ Most of the trials included patients with a minimum NIHSS score of 6 or 8, except MR CLEAN ${ }^{2}$ and EXTEND-IA ${ }^{3}$ studies. A pooled analysis of the major RCTs, the HERMES collaboration, indicated no benefit of endovascular treatment in patients with baseline NIHSS score between 0 to $5 .{ }^{9}$ On the other hand, two recent studies focused on low NIHSS score patients, suggested the efficacy of mechanical thrombectomy in this specific population. ${ }^{10-12}$ Currently, the natural history of patients with large vessel occlusion and low NIHSS score (LVOLN) is poorly known and yet to be determined. In this study, we aimed to evaluate efficacy and safety of mechanical thrombectomy in the treatment of acute stroke in LVOLN patients using new generation devices.

\section{Methods}

\section{Patient Population}

We analysed data extracted from the Italian Registry of Endovascular Treatment in Acute Stroke (Registro Endovascolare Italiano - REI), a multicenter, prospective, observational registry supported by the Italian Ministry of Health. ${ }^{13}$ The registry was approved by the local ethics committee. As a routine observational registry approved by the Italian Ministry of Health, a formal patient consent for registration was not required by Italian legislation. Enrolment was consecutive and prospectively recorded. Data collected included: patient's demographics, stroke risk factors, premorbid conditions, baseline stroke severity, baseline CT scan, non-invasive neurovascular imaging before treatment, endovascular treatment data and clinical follow-up. All the centres involved in the registry were asked to report all cases performed at their institution. Patient selection and interventional strategies were not pre-specified per protocol and were at the operator's discretion. All participating centers had safe implementation of treatments in stroke (SITS) accreditation for intravenous thrombolysis and neuroradiological expertise in the endovascular treatment of stroke. Data entry was performed in an electronic database by the stroke neurologist (clinical information) and by the interventional neuroradiologist (imaging and procedure details).

\section{Inclusion criteria}

We included adult patients (age $\geq 18$ years) with large vessel occlusion on the initial CTA/MRA and NIHSS $\leq 6$ on admission (or when the decision for the treatment was made) who underwent endovascular thrombectomy in the period from January 1, 2011 to December 31, 2015.

\section{Variables and outcome measurements}

We evaluated baseline characteristics (demographic data, stroke risk factors, baseline NIHSS score, site of occlusion, recanalization time), procedural and safety parameters (techniques and complication rate) and clinical outcomes (mRS at three months). Clinical outcome was correlated with: post-procedural mTICI, ischemic time, clot location, procedural complications and symptomatic intracranial hemorrhage. We used the ECASS classification to categorize hemorrhages. ${ }^{14}$

\section{Statistics}

Ordinal logistic regression (proportional odds model) was performed to analyse the relationship between 3month mRS scored as levels 0 to 6 , and baseline characteristics, ischemic time (categorized in $<4 \mathrm{~h}, 4-6 \mathrm{~h}$ and $>$ $6 \mathrm{~h}$ ), occlusion site, revascularization rate, complications, IV thrombolytic treatment, and age. Variables with skewed distributions were log (natural log) transformed in regression models and indicated as such in results. Ordinal logistic regression analysis took into consideration potential confounders of the association between ischemic time, independent variables and mRS (dependent variable), with the measure of association being the odds ratio. All regression models used a maximum likelihood algorithm to determine parameter estimates. All statistical analyses were performed using SAS software Version 9.4 (SAS Institute Inc., Cary, NC, USA), where differences were considered significant outside the $95 \%$ confidence interval (p 0.05).

\section{Results}

\section{Patient population and baseline characteristics}

A total of 134 of the 2826 patients (4.7\%) in the database met inclusion criteria. Baseline and clinical characteristics at admission are summarized in Table 1 . Ninety patients $(67.2 \%)$ had vessel occlusion in the anterior circulation, and 44 patients $(32.8 \%)$ presented with an occlusion in the posterior circulation. Overall, 116 patients were admitted directly to the tertiary hospital, and 18 were transferred from another institution. Previous tPA treatment was performed in $44(32.8 \%)$ patients: $26(59.1 \%)$ had bridging and $18(40.9 \%)$ had rescue treatments (i.e. MT was performed after failure of IV tPA).

Mean baseline NIHSS score was $4( \pm$ SD 1.86). Before endovascular treatment, inside the angiography suite, we observed a change in NIHSS from the baseline score in 45 patients (34\%): $37(28 \%)$ worsened whereas $8(6 \%)$ improved. The overall mean NIHSS change was +7 points; we did not find a significant association between NIHSS variation score and the occlusion site ( $p=0.57)$.

Endovascular treatment for LVO was performed in all but one patient who died before the intervention. All patients had a mTICI 0 or 1 on the first angiogram. 
Table 1. Baseline data of the patients.

\begin{tabular}{|c|c|c|}
\hline Variable & Mean & Median \\
\hline Age & $63.1(\mathrm{SD} 14.33)$ & $66(52-74)$ \\
\hline Gender $(\mathrm{M})$ & $54.5 \%(73 / 134)$ & \\
\hline Recent TIA/stroke & $10.3 \%(12 / 116)$ & \\
\hline Atrial fibrillation & $19 \%(22 / 116)$ & \\
\hline Diabetes & $15.5 \%(18 / 116)$ & \\
\hline Hypertension & $63 \%(73 / 116)$ & \\
\hline Heart Failure & $6 \%(7 / 116)$ & \\
\hline Smoking & $31.9 \%(37 / 116)$ & \\
\hline ICA stenosis & $6.7 \%(9 / 134)$ & \\
\hline Antiplatlets & $28.4 \%(38 / 134)$ & \\
\hline Anticoagulants & $13.4 \%(18 / 134)$ & \\
\hline $\begin{array}{l}\text { Anterior circulation } \\
\text { stroke }\end{array}$ & $67.2 \%(90 / 134)$ & \\
\hline \multicolumn{3}{|l|}{ Site of occlusion } \\
\hline Basilar artery & $28.3 \%(38 / 134)$ & \\
\hline$M 2$ & $22.3 \%(30 / 134)$ & \\
\hline M1 & $17.2 \%(23 / 134)$ & \\
\hline T siphon & $11.2 \%(15 / 134)$ & \\
\hline Tandem occlusion & $9 \%(12 / 134)$ & \\
\hline Extracranial ICA & $7.5 \%(10 / 134)$ & \\
\hline Vertebral artery & $3 \%(4 / 134)$ & \\
\hline$P 1$ & $1.5 \%(2 / 134)$ & \\
\hline Previous iv r-TPA & $32.8 \%(44 / 134)$ & \\
\hline Wake-up stroke & $8.2 \%(11 / 134)$ & \\
\hline Time to groin & 295' (SD135) & $305^{\prime}(55-790)$ \\
\hline Time to reperfusion & 344' (SD 147') & $330(90-860)$ \\
\hline $\begin{array}{l}\text { ASPECT score } \\
\text { on admission }\end{array}$ & $8.8($ SD 1) & \\
\hline \multicolumn{3}{|l|}{ Anesthesia } \\
\hline General* & $40.2 \%(45 / 112)$ & \\
\hline Local & $31.3 \%(35 / 112)$ & \\
\hline Neuroleptic & $28.6 \%(32 / 112)$ & \\
\hline \multicolumn{3}{|l|}{ NIHSS } \\
\hline NIHSS at admission & $4.2(3-6)$ & 5 \\
\hline $\begin{array}{l}\text { Worsening of } \\
\text { symptoms before } \\
\text { MT }\end{array}$ & $27.6 \%(37 / 134)$ & \\
\hline
\end{tabular}

*6 patients were shifted to general anesthesia due to clinical deterioration before the procedure.

\section{Procedural and technical outcomes}

Revascularization rates are reported in Table 2. Endovascular techniques were reported in 104 cases (78.2\%), divided as follows: stent-retriever alone in 70 (67.3\%) cases, aspiration alone in $22(21.2 \%)$ of the cases and a combination of aspiration and stent-retriever in 12 $(11.5 \%)$ patients. The mean number of passes per patient was 2.4..$^{1-15}$ Successful revascularization (mTICI 2b-3) was achieved in 98 patients $(73.7 \%)$.

In $33 / 133$ patients $(24.8 \%)$ a stent was deployed at the end of the procedure. Stents were deployed in the following locations: extracranial ICA $(N=15)$, extracranial vertebral artery $(N=7)$, basilar artery $(N=5)$, intracranial ICA $(N=2)$, M1 $(N=2)$, and M2 $(N=2)$. The decision to deploy
Table 2. Overall recanalization rate.

\begin{tabular}{lccc}
\hline & $\begin{array}{c}\text { Anterior } \\
\text { circulation }\end{array}$ & $\begin{array}{c}\text { Posterior } \\
\text { circulation }\end{array}$ & Total \\
\hline mTICI 0 & $6.7 \%(6 / 89)$ & $0 \%$ & $4.5 \%(6 / 133)$ \\
mTICI 1 & $5.6 \%(5 / 89)$ & $2.3 \%(1 / 44)$ & $4.5 \%(6 / 133)$ \\
mTICI 2a & $14.6 \%(13 / 89)$ & $22.7 \%(10 / 89)$ & $17.3 \%(23 / 133)$ \\
mTICI 2b & $13.5 \%(12 / 89)$ & $27.3 \%(12 / 44)$ & $18.1 \%(24 / 133)$ \\
mTICI 3 & $59.3 \%(53 / 89)$ & $47.7 \%(21 / 44)$ & $55.6 \%(74 / 133)$ \\
mTICI & $73 \%(65 / 89)$ & $75 \%(33 / 44)$ & $73.7 \%(98 / 133)$ \\
2b-3 & & & \\
\hline
\end{tabular}

an intracranial stent was driven by the presence of underlying stenosis in 7 cases and due to residual non-retrievable clot in 4 cases.

We counted 4 intraprocedural adverse events (3\%): 3 $(2 \%)$ subarachnoid hemorrhages caused by microwire perforation or by the stent-retriever and $1(0.7 \%)$ iatrogenic dissection. No embolizations in new territories were noted on final angiograms.

\section{Clinical outcomes}

Clinical outcome data are reported in Fig 1. At 90 days, 95/134 (70.9\%) had a good outcome (mRS0-2) and 39/134 (29.1\%) a poor outcome (mRS3-6). The overall rate of hemorrhage was $18 \%(24 / 133)$. The overall rate of symptomatic intracranial hemorrhage was 5.3\% (7/133). We did not find a significant difference in hemorrhagic transformation between patients with posterior and anterior circulation occlusion or previous administration of IV rt-PA. Table 3 shows the univariate analysis related to mRS grade shift at three months. Table 4 shows the univariate analysis of variables related to mRS score $0-2$ at three months.

All the patients who experienced a symptomatic intracranial hemorrhage within 24 hours had a poor outcome (7/7). No significant difference was noticed for poor clinical outcome in case of anterior or posterior circulation stroke. Regarding causes of mortality amongst patients who underwent thrombectomy, 13 (9.8\%) died from nonneurological related causes (5 pneumonia, 2 heart failure, 4 sepsis, 1 acute respiratory insufficiency, 1 died due to bowel infarction) and 3 due to intracranial hemorrhage. In two cases the cause of the death was not specified.

\section{Multivariable analysis}

Multivariable analysis showed that mTICI (0-2a vs $2 b-$ 3 ) and age (natural $\log$ ) were the only variables related to clinical outcomes (mTICI dichotomized: OR 2.60, $p=$ $0.018,1.235-5.45395 \% \mathrm{CL}$, age (natural $\log$ ): OR 1.35, $p=0.009,1.0251 .07595 \% \mathrm{CL}$ ). No other variables were associated with outcomes (IV t-PA, occlusion territory, side of occlusion, time from onset-to-groin-puncture). 


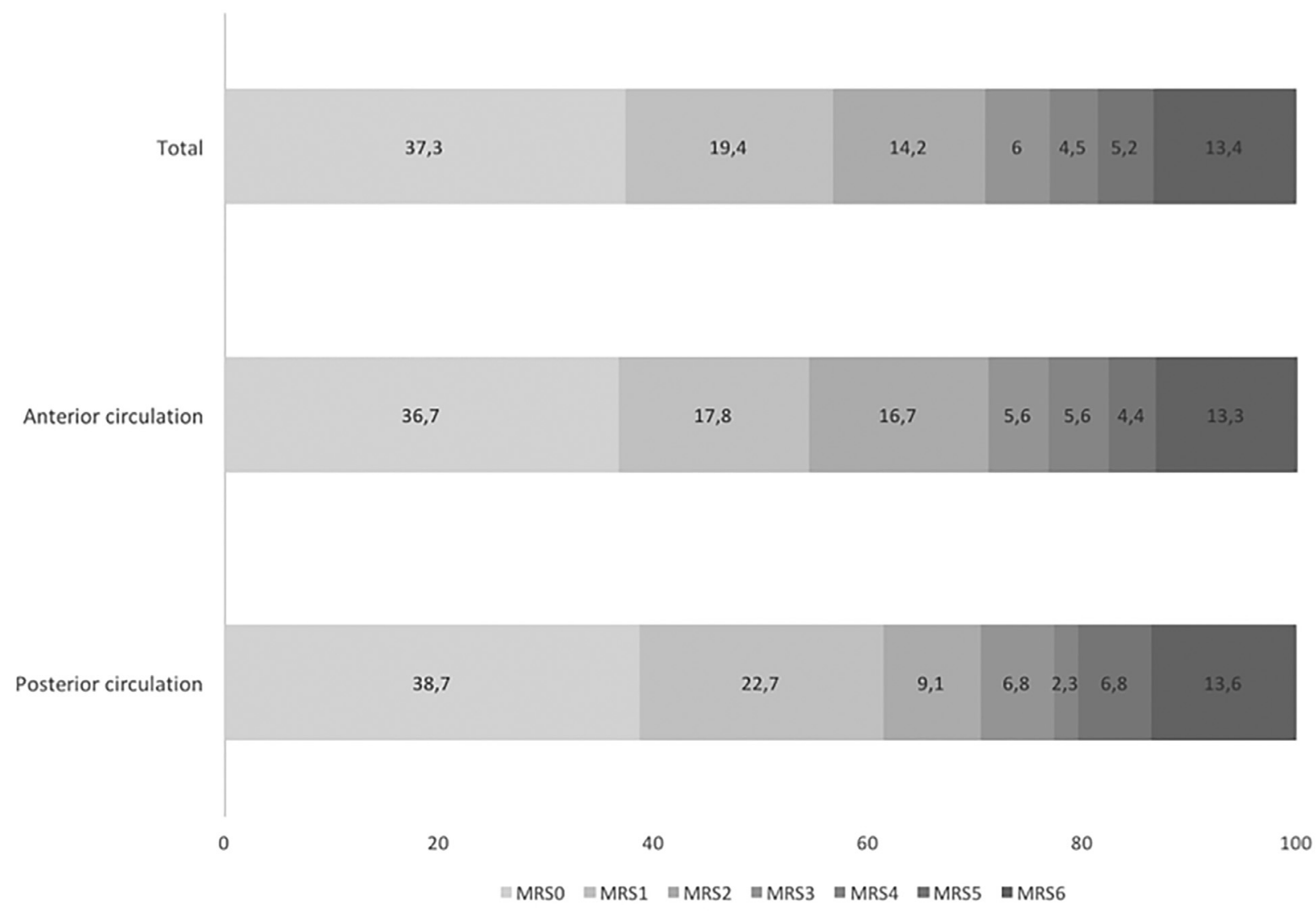

Fig. 1. Clinical outcome.

Table 3. Univariate analysis of variables related to $m R S$ grade shift at three months.

\begin{tabular}{llll}
\hline & $\mathrm{p}$ & OR & $\begin{array}{l}95 \% \text { wald } \\
\text { confidence } \\
\text { limits }\end{array}$ \\
\hline $\begin{array}{l}\text { TICI 0-2a } \\
\text { vs TICI 2b-3 }\end{array}$ & .005 & 2.70 & $1.34-5.42$ \\
Age* & .007 & 1.050 & $1.03-1.08$ \\
No IVtPA vs IVtPA & .054 & 1.93 & $0.99-3.78$ \\
Time to groin & .961 & 0.98 & $0.53-1.84$ \\
$\begin{array}{l}\text { Occlusion territory } \\
\quad \text { (AvsP) }\end{array}$ & .76 & 1.10 & $0.58-2.11$ \\
$\quad$ Side & .53 & 1.27 & $0.62-2.60$ \\
\hline
\end{tabular}

${ }^{*}$ Log Age

\section{Discussion}

Our study reports the results of mechanical thrombectomy in patients with large vessel occlusion and low NIHSS score in a large nationwide prospective registry. We observed that a good clinical outcome (mRS0-2) was achieved in $70.9 \%$ of the cases, and there were no differences in outcome between anterior and posterior circulation strokes presenting with low NIHSS. This datum is significantly higher than $46 \%$ reported by the HERMES
Table 4. Univariate analysis of predicting factors and their statistical significance per $m R S$ grade at three months cathegorized in.

\begin{tabular}{llll}
\hline & $\mathrm{p}$ & OR & $\begin{array}{l}95 \% \text { wald } \\
\text { confidence } \\
\text { limits }\end{array}$ \\
\hline $\begin{array}{l}\text { TICI 0-2a } \\
\text { vs TICI 2b-3 }\end{array}$ & .001 & 3.933 & $1.73-8.93$ \\
Age & .0001 & 1.072 & $1.04-1.11$ \\
No IVtPA vs IVtPA & .296 & 1.560 & $0.68-3.60$ \\
$\quad \begin{array}{l}\text { Time to groin } \\
\text { Occlusion territory }\end{array}$ & .257 & 0.984 & \\
$\quad$ (AvsP) & .937 & 0.969 & $0.44-2.14$ \\
Side & .968 & 0.948 & $0.40-2.25$ \\
\hline
\end{tabular}

*Log Age

meta-analysis. ${ }^{9}$ : the reason is probably due to the milder symptoms at the onset.

Based on our data, mechanical thrombectomy was relatively safe, as only $3 \%$ of patients had procedure-related complications- all of them without clinical significance. Revascularization and age were the main predictors of clinic outcomes.

Performing endovascular treatment in patients with mild symptoms is controversial due to the procedural risks 
and absence of clear data on the natural history of these patients. The MR CLEAN subgroup analysis, the HERMES meta-analysis (largely influenced by the data from MR CLEAN which included a majority of the low NIHSS score patients) and a recent multicentre cohort study did not report a benefit of endovascular treatment in the setting of low NIHSS stroke patients. ${ }^{2,9,15}$ On the other hand, other series and one meta-analysis reported an mRS0-2 at three months of $83-100 \%$ when patients were properly recanalized, similar to our findings. ${ }^{4,10,11,16-18}$

Regarding the natural history of low NIHSS score patients, previous reports showed good outcomes irrespective of treatment, because most patients have embolism in distal vessels, small vessel occlusion causing lacunar stroke, transient ischemic attack. ${ }^{19-22}$ However, when considering patients with LVO the natural history is different. Sato et al. analysed a subgroup of patients with documented LVO or large vessel stenosis ( $>50 \%$ ) on CTA or MRA and baseline NIHSS score $\leq 3$ and demonstrated that only $22 \%$ of these patients had good functional outcomes. ${ }^{23}$ Mokin et al. highlighted that $38 \%$ of patients with large vessel occlusion and mild symptoms on admission were not able to return home at discharge and had poor functional outcome. ${ }^{24}$ Such findings suggest that patients with LVOLN are prone to poor outcomes if recanalization is not achieved.

One of the interesting findings from our study was the fact that around $30 \%$ of our patients worsened from of neurological examination to the angio-suite, suggesting that the clinical status of these patients can rapidly change. We advance the hypothesis that LVOLN patients may have good collaterals which are temporarily sufficient to guarantee enough perfusion to the brain leading to mild neurological deficits, ${ }^{25}$ but are prone to collapse with time. It is unclear how and when the collateral circulation will collapse, with the most common hypotheses being variation in blood pressure and cerebral blood perfusion, ${ }^{26}$ increase of intracranial pressure caused by focal edema, ${ }^{27}$ venous steal phenomena ${ }^{28}$ or a combination of the previously mentioned processes. The inability to predict which patients will impair in the setting of LVOLN is a major impetus that favours treatment.

Good collaterals are probably the key of mild symptoms at onset and they are determined by multiple factors as genetics, environment and anatomy variants. We think that also the presence of a critical, long-standing atherosclerotic extracranial stenosis could play a role in the induction and proliferation of intracranial collateral circulation and the high number of patients who needed to be stented in our series seems to corroborate this hypothesis.

Our study has limitations. These data are from a single arm prospective study without a control group, and this does not allow us to compare directly the results of medical management to endovascular treatment. However, we noticed that around one out of three patients admitted with mild symptoms worsened before endovascular procedure, suggesting that a subset of LVOLN are clinically unstable and may rapidly deteriorate, thus supporting endovascular treatment.

The criteria to decide whether to treat or not to treat were not pre-specified in a protocol, which may imply a certain amount of heterogeneity in the decisional process in the participating centers. However, the REI is not a RCT but a real world experience database, thus better representing routine clinical practice.

There is no core lab adjudication for clinical and angiographic outcomes, and may have introduced some bias. However, angiographic and clinical outcomes were independently evaluated by the interventional neuroradiologist and a certified neurologist per study policy.

Future randomized controlled trials are needed to determine if endovascular treatment of LVO in low NIHSS patients is superior to medical management such as the MOSTE and MISTWAVE trials.

\section{Supplementary materials}

Supplementary material associated with this article can be found in the online version at doi:10.1016/j.jstrokecere brovasdis.2020.105091.

\section{References}

1 Saver JL, Goyal M, Bonafe A, et al. Stent-retriever thrombectomy after intravenous t-PA vs. t-PA alone in stroke. N Engl J Med 2015;372:2285-2295.

2 Berkhemer OA, Fransen PS, Beumer D, et al. A randomized trial of intraarterial treatment for acute ischemic stroke. N Engl J Med 2015;372:11-20.

3 Campbell BC, Mitchell PJ, Kleinig TJ, et al. Endovascular therapy for ischemic stroke with perfusion-imaging selection. N Engl J Med 2015;372:1009-1018.

4 Broderick JP, Berkhemer OA, Palesch YY, et al. Endovascular therapy is effective and safe for patients with severe ischemic stroke: pooled analysis of interventional management of stroke iii and multicenter randomized clinical trial of endovascular therapy for acute ischemic stroke in the Netherlands data. Stroke 2015;46:3416-3422.

5 Molina CA, Chamorro A, Rovira A, et al. REVASCAT: a randomized trial of revascularization with SOLITAIRE FR device vs. best medical therapy in the treatment of acute stroke due to anterior circulation large vessel occlusion presenting within eight-hours of symptom onset. Int J Stroke: Off J Int Stroke Soc 2015;10:619-626.

6 Goyal M, Demchuk AM, Menon BK, et al. Randomized assessment of rapid endovascular treatment of ischemic stroke. N Engl J Med 2015;372:1019-1030.

7 Jovin TG, Chamorro A, Cobo E, et al. Thrombectomy within $8 \mathrm{~h}$ after symptom onset in ischemic stroke. N Engl J Med 2015;372:2296-2306.

8 Bracard S, Ducrocq X, Mas JL, et al. Mechanical thrombectomy after intravenous alteplase versus alteplase alone after stroke (THRACE): a randomised controlled trial. Lancet Neurol 2016;15:1138-1147.

9 Goyal M, Menon BK, van Zwam WH, et al. Endovascular thrombectomy after large-vessel ischaemic stroke: a metaanalysis of individual patient data from five randomised trials. Lancet (Lond, Engl) 2016;387:1723-1731. 
10 Dargazanli C, Consoli A, Gory B, et al. Is reperfusion useful in ischaemic stroke patients presenting with a low national institutes of health stroke scale and a proximal large vessel occlusion of the anterior circulation? Cerebrovasc Dis (Basel, Switzerland) 2017;43:305-312.

11 Haussen DC, Bouslama M, Grossberg JA, et al. Too good to intervene? Thrombectomy for large vessel occlusion strokes with minimal symptoms: an intention-to-treat analysis. J Neurointervent Surg 2016.

12 Pfaff J, Herweh C, Pham M, et al. Mechanical thrombectomy in patients with acute ischemic stroke and lower NIHSS scores: recanalization rates, periprocedural complications, and clinical outcome. AJNR Am J Neuroradiol 2016.

13 Mangiafico S, Pracucci G, Saia V, et al. The Italian registry of endovascular treatment in acute stroke: rationale, design and baseline features of patients. Neurol Sci: Off J Italian Neurol Soc Italian Soc Clin Neurophysiol 2015;36:985-993.

14 Fiorelli M, Bastianello S, von Kummer R, et al. Hemorrhagic transformation within $36 \mathrm{~h}$ of a cerebral infarct: relationships with early clinical deterioration and 3-month outcome in the European Cooperative Acute Stroke Study I (ECASS I) cohort. Stroke 1999;30:2280-2284.

15 Sarraj A, Hassan A, Savitz SI, et al. Endovascular thrombectomy for mild strokes: how low should we go? Stroke 2018;49:2398-2405

16 Bhogal P, Bucke P, Ganslandt O, et al. Mechanical thrombectomy in patients with M1 occlusion and NIHSS score $</=5$ : a single-centre experience. Stroke Vasc Neurol 2016;1:165-171.

17 Nagel S, Bouslama M, Krause LU, et al. Mechanical thrombectomy in patients with milder strokes and large vessel occlusions. Stroke 2018;49:2391-2397.

18 Griessenauer CJ, Medin C, Maingard J, et al. Endovascular mechanical thrombectomy in large-vessel occlusion ischemic stroke presenting with low national institutes of health stroke scale: systematic review and meta-analysis. World Neurosurg 2018;110:263-269.

19 Smith EE, Fonarow GC, Reeves MJ, et al. Outcomes in mild or rapidly improving stroke not treated with intravenous recombinant tissue-type plasminogen activator: findings from get with the guidelines-stroke. Stroke 2011;42:3110-3115.

20 Ferrari J, Knoflach M, Kiechl S, et al. Early clinical worsening in patients with TIA or minor stroke: the Austrian stroke unit registry. Neurology 2010;74:136-141.

21 Ayis SA, Coker B, Rudd AG, et al. Predicting independent survival after stroke: a European study for the development and validation of standardised stroke scales and prediction models of outcome. J Neurol, Neurosurg, Psychiatry 2013;84:288-296.

22 Khatri P, Conaway MR, Johnston KC. Ninety-day outcome rates of a prospective cohort of consecutive patients with mild ischemic stroke. Stroke 2012;43:560-562.

23 Sato S, Uehara T, Ohara T, et al. Factors associated with unfavorable outcome in minor ischemic stroke. Neurology 2014;83:174-181.

24 Mokin M, Masud MW, Dumont TM, et al. Outcomes in patients with acute ischemic stroke from proximal intracranial vessel occlusion and NIHSS score below 8. J Neurointervent Surg 2014;6:413-417.

25 Mangiafico S, Saia V, Nencini P, et al. Effect of the interaction between recanalization and collateral circulation on functional outcome in acute ischaemic stroke. Intervent Neuroradiol: J Peritherap Neuroradiol, Surg Proced Relat Neurosci 2014;20:704-714.

26 Jiang B, Churilov L, Kanesan L, et al. Blood pressure may be associated with arterial collateralization in anterior circulation ischemic stroke before acute reperfusion therapy. J Stroke 2017.

27 Beard DJ, McLeod DD, Logan CL, et al. Intracranial pressure elevation reduces flow through collateral vessels and the penetrating arterioles they supply. A possible explanation for 'collateral failure' and infarct expansion after ischemic stroke. J Cereb Blood Flow Metab: Off J Int Soc Cereb Blood Flow Metab 2015;35:861-872.

28 Pranevicius O, Pranevicius M, Pranevicius H, et al. Transition to collateral flow after arterial occlusion predisposes to cerebral venous steal. Stroke 2012;43:575-579. 